TUM-HEP 244/96

SFB-375/96

hep-th/9604096

August 8, 2017

\title{
Post Inflationary Behaviour of String Moduli
}

\author{
André Lukas * ${ }^{\dagger}$, Alexander Niemeyer ${ }^{\ddagger}$ \\ and \\ Masahiro Yamaguchi $\S$ \\ Physik Department \\ Technische Universität München \\ D-85747 Garching, Germany
}

\begin{abstract}
We analyze the behaviour of moduli fields in string effective models between the end of inflation and reheating. The effective moduli potential during this era is derived for a class of simple models. We argue that this potential significantly stabilizes the modulus at its high energy minimum, if some restrictions on modular weights are met. Two mechanisms to further stabilize the moduli to their low energy minima are discussed explicitly: coinciding minima at a point of enhanced symmetry, and the smooth transition from high to low energy minimum by an effective mass term $C^{2} H^{2}$. For both cases we present explicit examples, and $C^{2}$ is found to be $O(10)$ at most. In addition, we show that during a smooth transition the reduction of the modulus amplitude strongly depends on the shape of the low energy potential.
\end{abstract}

\footnotetext{
*Email: alukas@physik.tu-muenchen.de

${ }^{\dagger}$ Address after March 1996: Department of Physics, University of Pennsylvania, Philadelphia, PA 19104, USA.

†Email: niemeyer@physik.tu-muenchen.de

$\S$ Email: myamaguc@physik.tu-muenchen.de

`On leave of absence from Department of Physics, Tohoku University, Sendai 980-77, Japan
} 


\section{Introduction}

In view of the current understanding of superstring theory, moduli fields are likely to have masses of the gravitino mass scale, which is assumed to be at, or at least not far from, the electroweak scale. The existence of such very weakly interacting scalar fields with an electroweak-scale mass would cause a cosmologically disastrous problem, which is referred to as the (cosmological) moduli problem in the context of string theory [1]. This problem can be regarded as a string version of the notorious Polonyi problem [2] associated with a hidden sector supersymmetry breaking in supergravity. The most severe consequence is that late moduli decay after coherent oscillation would totally change the abundances of light nuclei successfully predicted by the standard big-bang nucleosynthesis theory.

A number of ideas to solve the moduli problem (and the Polonyi problem) have been proposed over the last decade, some of which have been reviewed in ref. [3]. What makes the problem particularly serious is the fact that unlike most other unwanted relics the energy stored in moduli cannot be diluted away by ordinary inflation. At the end of inflation moduli will generically be displaced by $O(1)$ in Planck units from their low energy minimum and finally (once the Hubble constant approaches their mass) start late decaying coherent oscillations with the aforementioned disastrous consequences. Two types of (cosmological) solutions have been proposed in view of this particular problem. The first assumes a second late period of inflation [4] (e. g. at the weak scale) of a few e-foldings sufficiently long to dilute away the energy density stored in the moduli. A very interesting realization of this mechanism is provided by the idea of thermal inflation [5].

In this paper we will be concerned with the second type of solutions which attempts to stabilize the moduli to their minimum before the coherent oscillations start. A useful observation towards such a solution is that all fields receive an effective mass of the order of the Hubble parameter $H$ due to the energy density present in the universe [6]. This mass, which will dominate in early stages can stabilize moduli very effectively to a minimum. Unfortunately, this minimum - the high energy minimum - will generally not coincide with the low energy minimum and oscillations reappear as soon as the moduli start to roll towards the second. It has, however, been suggested that symmetries might provide a reason for the low and high energy minima to coincide [7].

In string theory the role of this symmetry can be played by modular invariance with the minimum sitting at a point of enhanced symmetry. In fact, a self-dual point is an extremum of the potential and the moduli problem can be solved once this extremum is arranged to be a minimum in each stage of the cosmological evolution. Though possible in principle, this scenario poses a problem when it is realized during the inflationary epoch: In models based on low energy effective string actions inflation is generally difficult to obtain [8] and it appears to be problematic to construct explicit examples as long as the precise mechanism of inflation is unknown. Not a single consistent example with an inflationary behaviour which stabilizes a modulus to a point of enhanced symmetry is known to us.

Recently Linde [9 has proposed a dynamical damping mechanism to reduce the am- 
plitude of the moduli which does not assume coinciding high and low energy minima. The crucial observation is that an effective moduli mass term $m_{T \text {,eff }} \simeq C^{2} H^{2} / 2$ with a large number $C^{2} \gg 1$ causes a significant damping of the oscillation amplitude. This happens because such a large value $C^{2}$ guarantees a 'smooth' transition of the modulus from the high to the low energy minimum at the time when $H$ has decreased down to the low energy modulus mass $m_{T}$ and the low energy potential becomes relevant. In ref. [9] it has been shown that this damping mechanism works for a quadratic low energy potential $V_{0} \simeq m_{T}^{2} T^{2} / 2$ and leads to a damping of the oscillation amplitude by a factor of $\sim \exp (-\pi p C / 2)$ where $p$ described the expansion rate of the universe via $H=p / t$.

In this paper, we would like to study the moduli potential in the post inflationary era with particular emphasis on the aforementioned possibilities to solve the moduli problem. In particular, we will derive the effective scalar potential for a modulus which describes its behaviour during the preheating phase after inflation, namely the era when the inflaton obeys a damped oscillation until it decays to reheat the universe. It is this era that determines the initial condition of the moduli oscillation. Therefore, for a solution of the moduli problem based on a symmetry induced minimum this era has to be considered necessarily. Moreover, we do not have to deal with consistency problems which arise from the unknown mechanism of inflation in string effective models if the stabilization of moduli is implemented in the preheating phase. All we have to assume is the existence of such a phase with the energy density being dominated by a coherently oscillating field which - depending on the type of inflation - might or might not coincide with the inflaton. Indeed, we will construct an explicit example which shows the stabilization of a modulus at a self-dual point during this era.

Also the mechanism of ref. [9] mentioned above can be addressed in our framework. A solution of the gravitino problem [10] implies a low reheating temperature $\lesssim 10^{9} \mathrm{GeV}$, corresponding to the Hubble parameter $\lesssim 1 \mathrm{GeV}$, well below the electroweak scale. Since the smooth transition between high and low energy minima occurs once $H$ drops to $H \sim m_{T}$ it will typically take place during the preheating era. In our framework, we are also able to explicitly compute the value $C^{2}$ which was put in by hand in ref. 90 and it can be checked whether it can be really made as large as required.

To illustrate the above ideas, we will concentrate ourselves on a simple class of models consisting of one modulus $T$ with a low energy potential $V_{0}$ and an oscillating field (inflaton) $\Phi$. They will be introduced in the next section where a general analysis of their properties during preheating is presented. In particular, we derive an effective modulus potential by performing a short time average over one period of the $\Phi$ oscillation. In section 3 we will focus on string motivated models: The low energy potential is assumed to be dual and to originate from gaugino condensation [11] and the (modulus dependent) Yukawa coupling of $\Phi$ is chosen in accordance with modular invariance. For these models we will first discuss the mechanism of stabilization at the high energy minimum in some detail. It turns out that this first stabilization (which is essential for the other mechanisms to work) is possible for a restricted range of modular weights of $\Phi$ only. Then we construct 
an explicit example with coinciding high and low energy minima at the self-dual point. As for Linde's mechanism, we first perform a 'phenomenological' analysis for $T$-dual low energy potentials. We find that the conclusions concerning a suppression of moduli oscillations can differ substantially from the case of a purely quadratic potential considered in ref. [9]. After this, information about the possible values $C^{2}$ is extracted. It turns out that large values are not associated with large but with steep couplings in the theory. In this respect the properties of modular functions naturally present in string effective models turn out to be very interesting. The results will be summarized and commented on in section 4 .

\section{The general framework}

In this section we would like to present a class of simple models suitable to describe the behaviour of moduli fields during the preheating period. We will investigate their general properties and in particular derive an effective moduli potential which incorporates the effect of the energy density stored in the coherent oscillations. Explicit examples will be given in the next section.

Our models consist of two chiral superfields, namely a modulus $T$ and the oscillating field $\Phi$. We do not restrict ourselves to any specific type of inflation which precedes the preheating phase. For chaotic inflation [12] $\Phi$ typically will be the inflaton itself whereas for hybrid inflation [13 a false vacuum expansion is followed by oscillations of $\Phi$.

The model is specified by a Kähler potential and a superpotential of the following form円:

$$
\begin{aligned}
& K=K_{0}(T, \bar{T})+Z(T, \bar{T})|\Phi|^{2} \\
& W=W_{0}(T)+\frac{1}{q} F(T) \Phi^{q}
\end{aligned} .
$$

Here the Kähler potential $K_{0}$ of the modulus and the metric $Z$ of $\Phi$ are kept general. In the examples we will concentrate on string motivated expressions for these quantities. Throughout this paper, we use Planck units $M \simeq 2.4 \times 10^{18} \mathrm{GeV}=1$. We have neglected higher $\Phi$ terms in eq. (1) which may dominate the potential during inflation, because we focus on the preheating phase where $\Phi<1$. For simplicity, we assume that the $\Phi$ field oscillates around the origin. The superpotential has been split into two parts: a modulus potential $W_{0}$ originating from nonperturbative effects like gaugino condensation and a $\Phi$-term with a modulus dependent coupling $F$. We allow the power $q$ to take the values $q=2,3$ corresponding to a mass term or to a Yukawa coupling for $\Phi$. Let us denote the intrinsic mass scales in $W_{0}$ by $m_{T}=O\left(W_{0}\right)$ and in $F$ by $M_{\Phi}=O(F)$. To be consistent with the constraint on the anisotropy of the photon background the mass (coupling) $M_{\Phi}$ should be well below the Planck scale, typically $M_{\Phi} \simeq 10^{-5}-10^{-6}$ for chaotic inflation. For hybrid inflation, $M_{\Phi}$ can be taken somewhat smaller. We will assume throughout this discussion that the low energy modulus mass $m_{T}$ is much smaller, i. e. $m_{T} \ll M_{\Phi}$. For

\footnotetext{
${ }^{1}$ Chiral superfields and their scalar components will be denoted by the same symbol.
} 
moduli potentials based on gaugino condensation we have $m_{T} \sim m_{3 / 2} \sim 10^{-15}$ so that this can be safely assumed.

Generally, the behaviour of the model (1) is expected to be complicated. It has to be analyzed in the full field space of four real fields and using the complete scalar potential. Having the ordinary scenario of reheating in mind one might think about the following situation: A rapidly oscillating $\Phi$ dominates the energy density of the universe and the modulus is moving in this given 'background'. Then an effective potential for the modulus can be obtained by a short time average over one period of the $\Phi$ oscillation. Such an average procedure would simplify the situation considerably. Let us now analyze under which assumptions this picture is really correct.

As soon as $\Phi \ll 1$ the scalar potential can be expanded as

$$
\begin{aligned}
V & \simeq V_{0}+\tilde{V}_{1} \\
\tilde{V}_{1} & =f(T, \bar{T})|\Phi|^{2 q-2} \\
f & =Z^{-1} e^{K_{0}}|F|^{2},
\end{aligned}
$$

where $V_{0}$ is the low energy modulus potential originating from $W_{0}$. Higher powers in $\Phi$ have been neglected. First we should require that the energy density is dominated by $\Phi$, i. e. $V_{0} \ll \tilde{V}_{1}$. Then $H^{2} \sim \tilde{V}_{1}$ and the condition is consistently fulfilled as long as

$$
H^{2} \gg V_{0} \sim m_{T}^{2}
$$

Since the low energy mass $m_{T}$ is much smaller than the inflationary scale $M_{\Phi}$ this condition will be fulfilled for a wide range in $H$. Even for $H \lesssim m_{T}$ it may hold if $T$ is close enough to its low energy minimum. We assume that this is the case in view of the fact that we are concerned with a solution of the cosmological moduli problem where the amplitude of the moduli oscillation is either small or damped quickly. Self-consistency of this assumption should be checked in each case. From the expressions (2) we can estimate the effective masses for $T$ and $\Phi$ as follows:

$$
\begin{aligned}
& m_{T, \text { eff }}^{2}=\left|\frac{f_{T}}{f}\right|^{2} H^{2} \\
& m_{\Phi, \text { eff }}^{2}=\frac{H^{2}}{\left|\Phi^{2}\right|},
\end{aligned}
$$

with

$$
\frac{f_{T}}{f}=-\frac{Z_{T}}{Z}+K_{0 T}+\frac{F_{T}}{F} .
$$

A short time average is justified if $m_{T \text {,eff }} \ll m_{\Phi, \text { eff }}$ which in turn implies

$$
|\Phi| \ll\left|\frac{f}{f_{T}}\right| .
$$


Inspection of eq. (6) shows that this condition is usually fulfilled once $|\Phi|$ is sufficiently small. However there is one important exception: The quantity $f_{T} / f$ can become very large close to zeros $F(T)=0$ of the Yukawa coupling. For the following derivation we therefore assume that the system - at least for a certain period after $|\Phi|$ became small does not evolve towards such a point or, equivalently, that the coupling strength $|F|$ is bounded from below in the relevant part of field space. Later on, we will comment on what happens if this condition is violated.

Oscillation of $\Phi$ will dominate the energy density of the universe as long as $H=p / t \sim$ $\Gamma_{\Phi} \sim T_{\mathrm{RH}}^{2}$ where $H=\dot{a} / a$ is the Hubble parameter, $\Gamma_{\Phi}$ is the decay constant of $\Phi$ and $T_{\mathrm{RH}}$ is the reheating temperature. For a radiation like behaviour of $H$ we have $p=1 / 2$, whereas a matter like behaviour is characterized by $p=2 / 3$. In view of the gravitino problem $T_{\mathrm{RH}} \lesssim 10^{9} \mathrm{GeV}$. This implies that the universe can be dominated by $\Phi$ oscillations down to values of $H \lesssim 1 \mathrm{GeV}$. In particular the start of the coherent modulus oscillations in the low energy potential responsible for the moduli problem at $H \sim m_{T}$ can fall into this era.

Let us now carry out the averaging procedure to obtain an effective potential for the modulus in the background of the oscillating field $\Phi$. As discussed, we assume that the conditions (3) and (7) hold. The system is described by a Lagrange function

$$
\mathcal{L}=a^{3}\left[-\left(\frac{\dot{a}}{a}\right)^{2}+K_{i \bar{k}} \dot{\phi}^{i} \dot{\phi}^{\bar{k}}-V\right]
$$

with the Kähler metric $K_{i \bar{k}}=\partial_{i} \partial_{\bar{k}} K$ and the fields denoted by $\phi^{i}$. For simplicity, we have assumed a flat Robertson-Walker universe. The conjugate momenta are given by

$$
\pi_{i}=a^{3} K_{i \bar{k}} \dot{\bar{\phi}}^{\bar{k}}
$$

and the equations of motion can be written in the form

$$
\frac{d}{d t}\left(K_{i \bar{k}} \dot{\phi}^{i}\right)+3 H K_{i \bar{k}} \dot{\phi}^{i}-\left(\partial_{\bar{k}} K_{i \bar{m}}\right) \dot{\phi}^{i} \dot{\bar{\phi}}^{\bar{m}}+\partial_{\bar{k}} V=0
$$

Sometimes it is useful to normalize the second derivative term in eq. (10) to get

$$
\ddot{\phi}^{i}+3 H \dot{\phi}^{i}+\Gamma_{j k}^{i} \dot{\phi}^{i} \dot{\phi}^{j}+\partial^{i} V=0,
$$

with the Kähler connection $\Gamma$ defined by $\Gamma_{i j}^{k}=K^{k \bar{l}} \partial_{i} K_{j \bar{l}}$. Furthermore, the Friedmann equation reads

$$
H^{2}=K_{i \bar{k}} \dot{\phi}^{i} \dot{\phi}^{\bar{k}}+V
$$

A straightforward computation under the above assumptions and using eq. (10) leads to

$$
\frac{d}{d t}\left(\pi_{\Phi} \Phi\right) \simeq a^{3}\left(U-(q-1) \tilde{V}_{1}\right)
$$


where $U=K_{i \bar{k}} \dot{\phi}^{i} \dot{\bar{\phi}}^{\bar{k}}$ is the kinetic energy. The short time average (which we denote by $<. .>$ in the following) of $d\left(\pi_{\Phi} \Phi\right) / d t$ approximately vanishes and we have

$$
<U>\simeq(q-1)<\tilde{V}_{1}>\text {. }
$$

With the energy density and the pressure $\rho=\langle U+V\rangle, P=\langle U-V\rangle$ we derive an equation of state

$$
P=\nu \rho, \quad \nu=\frac{q-2}{q} .
$$

and the Hubble parameter behaves like

$$
H=\frac{p}{t}, \quad p=\frac{2}{3(1+\nu)}=\frac{q}{3 q-3} .
$$

As expected, for $q=2$ (quadratic $\Phi$ potential) the expansion corresponds to a matter dominated universe and for $q=3$ (quartic $\Phi$ potential) it corresponds to a radiation dominated universe. Since $<\tilde{V}_{1}>=H^{2} / q$ we can carry out the following replacement in the EOM for $T$

$$
<\frac{\partial \tilde{V}_{1}}{\partial \bar{T}}>=\frac{1}{q} \frac{f_{\bar{T}}}{f} H^{2} .
$$

This leads to the effective EOM for $T$ during preheating

$$
\ddot{T}+3 H \dot{T}+\frac{K_{0 T T \bar{T}}}{K_{0 T \bar{T}}} \dot{T}^{2}+K_{0 T \bar{T}}^{-1} \frac{\partial V_{\text {eff }}}{\partial \bar{T}}=0,
$$

with the potential $V_{\text {eff }}$ given by

$$
\begin{aligned}
& V_{\text {eff }}=V_{0}+V_{1}=V_{0}+\frac{1}{q} g H^{2} \\
& g=\ln f=-\ln Z+K_{0}+\ln |F|^{2} .
\end{aligned}
$$

For $H \gg m_{T}$ the potential (19) is dominated by the second term originating from the $\Phi$ oscillations. The modulus will therefore settle down to one of the minima $T_{1}$ of $g$. This value can be assumed as an initial value for the further development of $T$ which sets in once the low energy potential becomes important, i. e. once $H$ approaches $m_{T}$. In the spirit of ref. [9] we can introduce a parameter $C^{2}$

$$
\begin{aligned}
C^{2}(T) & =\frac{1}{q} \frac{g_{\bar{T}}}{T-T_{1}} \\
g_{T} & =-\frac{Z_{T}}{Z}+K_{0 T}+\frac{F_{T}}{F} .
\end{aligned}
$$

Then the EOM can be rewritten as

$$
\ddot{T}+3 H \dot{T}+\frac{K_{0 T T \bar{T}}}{K_{0 T \bar{T}}} \dot{T}^{2}+K_{0 T \bar{T}}^{-1}\left(\frac{\partial V_{0}}{\partial \bar{T}}+C^{2}(T) H^{2}\left(T-T_{1}\right)\right)=0 .
$$


Notice that, in contrast to ref. [9], $C^{2}$ depends on $T$ and can - along with the starting value $T_{1}$ - be computed explicitly once a specific model has been chosen.

Let us now discuss the general consequences of this result before we turn to concrete models. Of course it might happen that the system runs into a region of very low coupling $|F(T)|$ even before the above stage is reached, i. e. before $|\Phi|$ is sufficiently small. In this case which strongly depends on the initial conditions there is no chance to generate a substantial damping of the modulus and we are left with the usual moduli problem.

If however the system evolves according to the above scenario, a damping of the modulus oscillation might result. First we would like to analyze the stabilization of $T$ to the high energy minimum $T_{1}$. Clearly, $g_{T}\left(T_{1}\right)=0$, and for $T_{1}$ to be a stable minimum the requirement

$$
g_{T \bar{T}}\left(T_{1}\right)>\left|g_{T T}\left(T_{1}\right)\right|
$$

has to be fulfilled. In particular $g_{T \bar{T}}>0$, which might impose severe restrictions on the model since $g_{T \bar{T}}$ does not depend on the superpotential but on the Kähler potential only. The stability condition (22) can be used to derive an upper bound on the value of $C_{1}^{2}=C^{2}\left(T_{1}\right)$ which determines how effective the stabilization to $T_{1}$ can be. From eq. (20) we have

$$
C^{2}\left(T \simeq T_{1}\right) \simeq \frac{1}{q}\left[g_{T \bar{T}}\left(T_{1}\right)+\frac{\bar{T}-\bar{T}_{1}}{T-T_{1}} g_{T T}\left(T_{1}\right)\right]
$$

and with condition (22) it follows that

$$
\left|C_{1}^{2}\right| \leq \frac{2}{q} g_{T \bar{T}}\left(T_{1}\right)
$$

Moreover, the order of $\left|C_{1}^{2}\right|$ will be given by the RHS of eq. (24). Notice that again this value depends on the Kähler potential of the theory only. The implications of eqs. (22) and (24) for string motivated models and the stabilization process will be studied in more detail in the next section.

For the moment we assume that $T$ has been driven to $T_{1}$ with a certain precision and starts its further evolution from this point. If $T_{1}$ coincides with the low energy minimum $T_{0}$ the stabilization is definite and moduli oscillations are suppressed.

Suppose that $T_{1}$ and $T_{0}$ do not coincide. Then, once $H \sim m_{T}$ the modulus will feel the low energy potential and will start oscillations around the true minimum $T_{0}$. The moduli problem simply reappears. In ref. [9] it has been shown that assuming a quadratic low energy potential these oscillations can be suppressed substantially for large values of $C^{2}$ by a factor of order $\exp (-\pi p C / 2)$. This mechanism comes into operation as soon as $H$ drops to $H \sim m_{T}$. Therefore, it may play a role for the models considered here if $T_{\mathrm{RH}} \lesssim m_{T}^{1 / 2}$ so that domination of the $\Phi$ oscillations is maintained sufficiently long. Such a low value of the reheating temperature is also required in order to solve the gravitino problem. 
Large values of $C^{2}$ cannot be obtained by simply introducing a large coupling in the theory. As eq. (20) shows a coupling constant in front of $F$ or $Z$ simply drops out. Instead, what one needs is a very steep coupling such that $F_{T} / F$ becomes large. Since generally $C^{2}$ is proportional to $V^{\prime} / V$ the validity of this statement is not restricted to our particular class of models. In the next section we will discuss how the conclusions of ref. [9] change for $T$-dual low energy potentials and to what extent large values of $C^{2}$ can be obtained in string motivated models.

Finally, the modulus might run into a region with vanishing Yukawa coupling $|F(T)|$ even after our effective potential description becomes valid. As can be seen from eq. (19) these points show up in the effective potential as singularities $V_{\text {eff }} \rightarrow-\infty$. If such zeros exist the system might therefore easily be attracted to them. To analyze the qualitative behaviour close to such a point $T_{2}$ let us expand $F \simeq F_{1}^{\prime}\left(T-T_{2}\right)$. Then, according to the condition (7) our averaging procedure breaks down once $1 / C^{2} \sim\left|T-T_{2}\right| \sim|\Phi|$ and $T$ will start to oscillate around $T_{2}$ with an initial amplitude of $|\Phi|$. Since $|\Phi|$ can be quite small when this happens a large suppression of the $T$ amplitude might result. Again, this guarantees a (at least partial) solution of the moduli problem only if $T_{2}$ coincides with the low energy minimum $T_{0}$.

For comparison we would like to briefly mention the requirements on the amplitude of the coherent moduli oscillation posed by the nucleosynthesis constraint. The energy density $\rho_{T}$ of these oscillations at the time of the decay of $T$ divided by the photon density $n_{\gamma}$ is given by

$$
\frac{\rho_{T}}{n_{\gamma}} \simeq T_{\mathrm{RH}} T_{\mathrm{in}}^{2},
$$

$T_{\text {in }}$ being the initial oscillation amplitude and assuming a matter dominated preheating phase.

In order not to dissociate light nuclei this quantity should be less than typically $10^{-8}$ to $10^{-12} \mathrm{GeV}$ (for ordinary moduli masses). For a reheating temperature of $T_{\mathrm{RH}}=10^{9}$ $\mathrm{GeV}$ this is satisfied, if $T_{\mathrm{in}} \lesssim 10^{-9}$ to $10^{-11}$ in Planck units.

In the case of a quadratic low energy potential as used in [9] one therefore needs values of $C^{2} \gtrsim 10^{2}-10^{3}$.

\section{Applications and examples}

In this section we would like to discuss the various possibilities mentioned above more explicitly. We will concentrate on string motivated models specified by

$$
K_{0}=-w \ln (T+\bar{T}), \quad Z=\frac{1}{(T+\bar{T})^{n}} .
$$

Usually, for a modulus we have $w=3$. The modular weight of $\Phi$ is denoted by $n$, i. e. under $T \rightarrow 1 / T$ duality $\Phi$ transforms as $\Phi \rightarrow \Phi / T^{n}$. To have a duality invariant 
theory the modular weight $m$ of the Yukawa coupling $F$ should be given by $m=w-q n$. Application of eq. (19) leads to an effective potential

$$
g=(n-w) \ln (T+\bar{T})+\ln |F|^{2},
$$

and the quantity $g_{T}$ which determines the value of $C^{2}$ is given by

$$
g_{T}=\frac{n-w}{T+\bar{T}}+\frac{F_{T}}{F} .
$$

The potential $g$ is modular invariant if and only if $\Phi$ has a vanishing weight $n=0$. Since $\Phi$ has been 'integrated' out to arrive at the above result this property could be expected.

The explicit form of Yukawa couplings in string theory has been computed in a number of publications [14, 15]. Generally, it has been found to be consistent with modular invariance. As an illustrative class we will consider couplings of the form

$$
F(T) \Phi^{q}=M_{\Phi} \frac{j^{l}(T)}{\eta^{2 m}(T)} \Phi^{q}
$$

with the Dedekind function $\eta$ and the totally modular invariant function $j$. By symmetry requirements $F$ could contain an arbitrary function of $j(T)$. Here we study only the subclass (29) with an arbitrary power $l$ of $j$. Yukawa couplings containing $j$-functions show up for example in Calabi-Yau compactifications [16]. In orbifold models the modular weight $n$ of $\Phi$ has been found to be in the range [17]

$$
10 \geq n \geq-4
$$

Furthermore, we concentrate on a specific form of the low energy moduli potential, which is related to the potential one expects from the process of $\mathrm{T}$-dual gaugino condensation [18]. Assuming gaugino condensation in a model consisting of dilaton $S$ and modulus $T$ in the usual way, i.e.

$$
\begin{aligned}
K_{0} & =-\ln (S+\bar{S})-3 \ln (T+\bar{T}) \\
W_{0} & =\Omega(S) / \eta(T)^{6}
\end{aligned}
$$

one arrives at the scalar potential

$$
V_{0}(S, T)=\frac{1}{T_{R}^{3}\left|\eta(T)^{12}\right|}\left(A(S)+B(S)\left|2 \frac{T_{R} \eta^{\prime}(T)}{\eta(T)}+1\right|^{2}\right) .
$$

where $T_{R}=T+\bar{T}$. The functions $A(S)$ and $B(S)$ are given in terms of $\Omega(S)$. Usually $A(S)=-B(S)(B(S) \geq 0$ always) at the minimum (supersymmetry is not broken by the dilaton, but by the modulus) and this will give the famous value $T \approx 1.23$. The shape of this potential is shown in fig. 1 . 


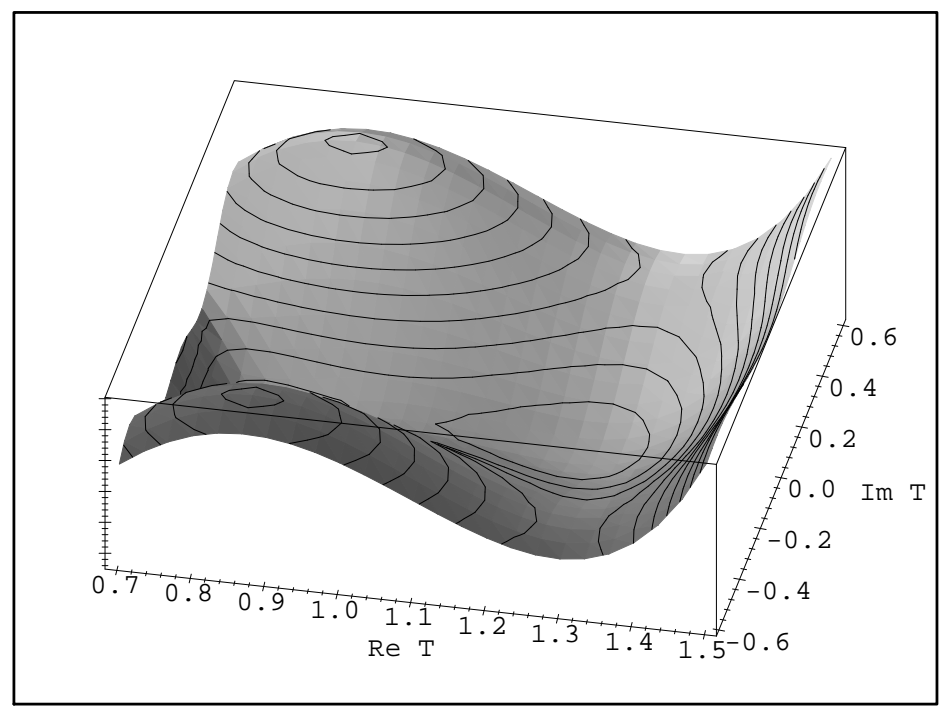

Fig 1: Gaugino condensation potential with minimum at $T=1.23$

If $B(S)=0$, which corresponds to unbroken supersymmetry in the $T$-direction the minimum will be at $T=\rho$ (the other self-dual point with $\rho=\exp (i \pi / 3)$ ). This implies that supersymmetry is unbroken by this condensation process since for the factorizing Ansatz (32) supersymmetry cannot be broken by the dilaton. The potential looks like in fig. 2 .

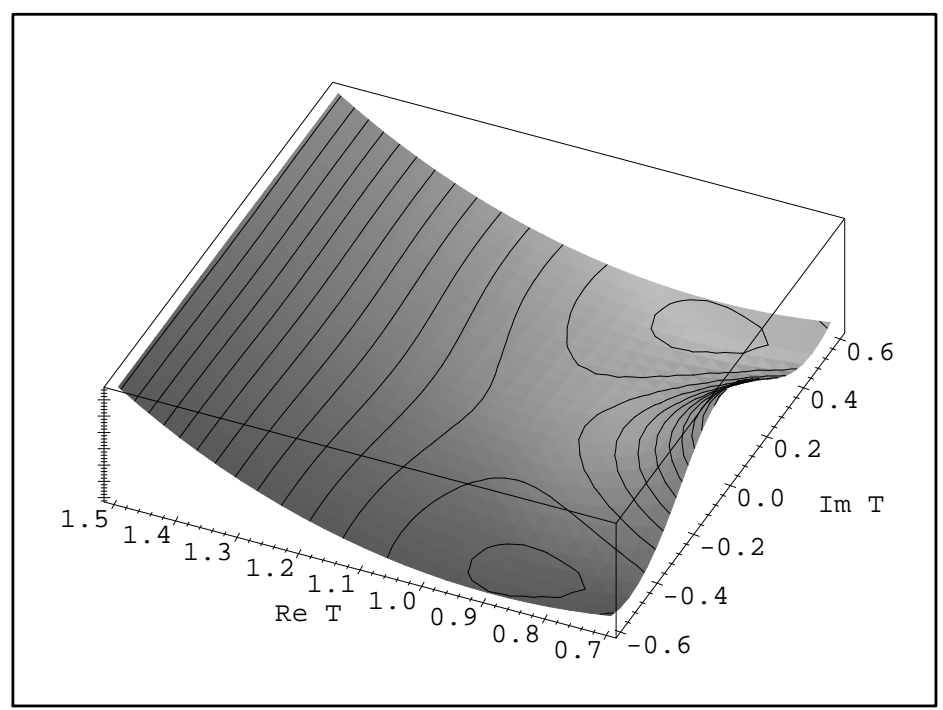

Fig 2: Gaugino condensation potential with minimum at $T=\rho$

A stabilization of the dilaton is usually difficult to achieve [19] and we will not address this question here. Instead, to keep our models as simple as possible we will consider only the $T$-dependence of the potential. Consequently, we assume that the dilaton VEV is fixed by some unknown mechanism and $A(S), B(S)$ are thus determined. 
Of course it is well known that the potentials discussed here generically have a negative cosmological constant. Sometimes one can shift the vacuum energy to zero by adjusting the value of an additional constant term in the superpotential. We simply assume that the potentials we study are shifted in such a way that the cosmological constant vanishes.

\subsection{Stabilization at the high energy minimum}

Now that we have specified our models let us apply the general results of the previous section. First we study the stabilization to the high energy minimum $T_{1}$, which is essential for any further suppression of moduli oscillations. As we have seen $T_{1}$ is a stable minimum of the high energy potential provided eq. (22) holds. Since

$$
g_{T \bar{T}}=\frac{w-n}{(T+\bar{T})^{2}},
$$

this implies as a necessary (but not sufficient) condition that

$$
n<w=3 \text {, }
$$

which constitutes a significant restriction on the modular weight $n$ of $\Phi$. If this bound is violated, no stabilization at the high scale and consequently no further stabilization can take place. Furthermore, from eq. (24) we have

$$
\left|C_{1}^{2}\right| \leq \frac{2}{q} \frac{|w-n|}{\left(T_{1}+\bar{T}_{1}\right)^{2}} .
$$

Given the bound (30) on the modular weight and the fact that the starting value $T_{1}$ will be typically $O(1)$ this restricts $C_{1}^{2}$ to a few at most. In view of this constraint it seems unlikely that the moduli problem can be solved completely by the ' $C^{2}$-mechanism' of ref. [9] though a larger value of $C^{2}(T)$ at $T \neq T_{1}$ cannot be excluded. We will come back to this point later on.

The stabilization of $T$ to $T_{1}$ is governed by the EOM 1 (cf. eq. (21))

$$
\ddot{T}+3 H \dot{T}+\frac{\left(T_{1}+\bar{T}_{1}\right)^{2}}{w} C_{1}^{2} H^{2}\left(T-T_{1}\right) \simeq 0 .
$$

Note that we have omitted the low energy potential $V_{0}$ which is unimportant at this early stage. The solutions of eq. (36) can be written as $\left(T-T_{1}\right) \sim t^{-\alpha}$ with

$$
\alpha=\frac{3 p-1}{2} \pm \sqrt{\left(\frac{1-3 p}{2}\right)^{2}-\frac{p^{2}\left(T_{1}+\bar{T}_{1}\right)^{2}}{w^{2}} C_{1}^{2}} .
$$

Damping of the $T$ oscillations

$$
\frac{\Delta T_{\mathrm{f}}}{\Delta T_{\mathrm{i}}}=\left(\frac{t_{\mathrm{i}}}{t_{\mathrm{f}}}\right)^{\alpha} \sim\left(\frac{m_{T}}{M_{\Phi}}\right)^{\alpha}
$$

\footnotetext{
${ }^{2}$ The term proportional to $\dot{T}^{2}$ in eq. (21) can be consistently neglected.
} 
will be most effective if the square root in eq. (37) is purely imaginary which is the case for a real and moderately large $C_{1}^{2} \sim O(1)$. Consequently $\alpha$ is bounded by

$$
\alpha \leq\left\{\begin{array}{l}
\frac{1}{2} \text { for } p=\frac{2}{3} \\
\frac{1}{4} \text { for } p=\frac{1}{2}
\end{array}\right.
$$

and typical maximal damping ratios are $10^{-5}$ and $10^{-2.5}$ for $p=2 / 3$ and $p=1 / 2$ respectively (for $M_{\Phi} \sim 10^{-5}$ and $m_{T} \sim 10^{-15}$ ). Though this suppression itself is definitely not sufficient to solve the moduli problem for $\Delta T_{\mathrm{i}} \sim O(1)$ it significantly improves a preexisting suppression $\Delta T_{\mathrm{i}} \ll 1$ originating from the inflationary epoch. Of course one has to make sure that no destabilization occurs in the following evolution which is the problem we are going to address now.

\subsection{High and low energy minima coincide}

Models in which the minima of the high energy potential and the low energy potential coincide, are very attractive from the viewpoint of the cosmological moduli problem. In this case the modulus will be fixed at this minimum at early times, when the curvature at the minimum is large as discussed in the previous subsection. During the transition to the low energy potential it will safely stay at that point.

In principle, this situation can be achieved by carefully tuning parameters in the potential. Such a choice would be neither natural nor stable under radiative corrections and is clearly unreasonable. Therefore, coincidence of low and high energy minima should be guaranteed by a symmetry. In a string framework duality is a very natural candidate for this symmetry which has been proposed in ref. [7].

For our models, this can be realized for $n=0$ only since otherwise duality is broken by the effective potential. This fixes the modular weight of the Yukawa coupling $F$ to $m=w=3$. Then, by modular invariance low and high energy potential both possess extrema at 1 and $\rho=\exp (i \pi / 3)$. For a successful example, one of these points should be arranged to be a minimum for both contributions to the potential.

For $T_{1}=\rho$ we can make a more detailed prediction of the stabilization at the high energy minimum. The second term in eq. (23) vanishes at $T_{1}=\rho$ and the value of $C^{2}$ at this high energy minimum can be explicitly computed to be

$$
C_{1}^{2}=\frac{1}{q} \frac{w}{\left(T_{1}+\bar{T}_{1}\right)^{2}}=\frac{1}{q} .
$$

According to eq. (37) this implies for the power $\alpha$

$$
\alpha=\left\{\begin{array}{c}
\frac{1}{3} \text { for } p=\frac{2}{3} \\
\frac{1}{4} \text { for } p=\frac{1}{2}
\end{array},\right.
$$

corresponding to a typical damping of $10^{-3.3}$ and $10^{-2.5}$ for $p=2 / 3$ and $p=1 / 2$, respectively. 
We start with an example for a minimum at $\rho$. A simple Yukawa coupling with weight $m=3$ is given by

$$
F=\frac{M_{\Phi}}{\eta^{6}(T)} .
$$

The resulting effective potential $g$ has a minimum at $T_{1}=\rho$. As discussed above, the low energy minimum of $V_{0}$ is at $\rho$ if supersymmetry is unbroken (because then $B(S)=0$ ).

A model where the minima coincide at the other self dual point $T=1$ can be constructed as well. A Yukawa-coupling

$$
F=\frac{M_{\Phi} j^{l}(T)}{\eta^{6}(T)}, \quad-0.068>l>-0.016
$$

will give the required minimum of $g$ at $T=1$. For a superpotential of the form $W_{0}=$ $\Omega(S) /\left(\eta^{6}(T) j(T)\right)$ instead of $W_{0}=\Omega(S) / \eta^{6}(T)$ the low energy minimum in $T$-direction will be also at $T=1$. The low energy potential of this model looks like in fig. 3 .

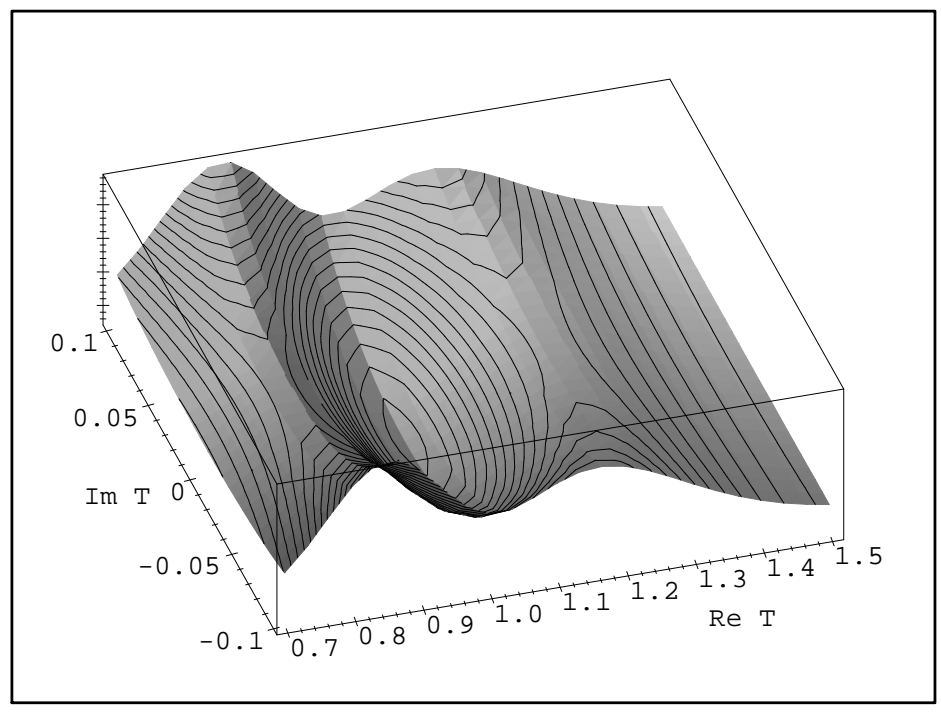

Fig 3: Potential for $W_{0}=\Omega(S) /\left(\eta^{6}(T) j(T)\right)$

Although explicit orbifold calculations do not lead to $j$-functions [15], this superpotential is perfectly consistent with duality and might originate from Calabi-Yau compactifications, where similar moduli dependencies show up [16].

Clearly, stabilization of a modulus at one of its self-dual points implies unbroken supersymmetry in this direction. Thus, if supersymmetry is broken by a modulus at all at least this modulus cannot be stabilized in this way.

\subsection{High and low energy minima do not coincide}

The situation described above may not be expected for all the (numerous) moduli of a superstring theory. Most probably not for each modulus the minima of the low and high 
energy potentials will coincide. We should therefore study this case and analyze, whether the mechanism described in [9] will sufficiently damp the coherent oscillations during the transition of the modulus from $T_{1}$ to $T_{0}$ (its final minimum). First we will generalize the approach of [9] to a more realistic low energy potential, but keep the high energy potential quadratic. As we will see, this will already change the results significantly.

\subsubsection{High energy minimum coincides with maximum of low energy potential}

A smooth transition does not take place at all if the high energy minimum coincides with a low energy maximum.

At this point we have $V_{0}^{\prime}\left(T_{1}\right)=V_{1}^{\prime}\left(T_{1}\right)=0$ and $V_{0}^{\prime \prime}>0, V_{1}^{\prime \prime}<0$. It is clear that at all times $V_{\text {eff }}^{\prime}\left(T_{1}\right)=0$ and that at some point of time $V_{\text {eff }}^{\prime \prime}\left(T_{1}\right)=0$. Therefore the position of the minimum of $V_{\text {eff }}$ will not move smoothly from the initial to the final low energy minimum with increasing time. Instead, the modulus will be released from its minimum at $T_{1}$ at the time when $V_{\text {eff }}^{\prime \prime}\left(T_{1}\right)=0$. Thus no reduction of the coherent oscillation amplitude can result, regardless of how strong the high energy potential is (i.e. regardless of the value of $C^{2}$ ). The mechanism of [9] cannot work in this case.

One can show that this situation occurs not only if the early minimum and the late maximum coincide, but also when they are close to each other. The maximal separation of the two extrema depends of course on the shape of the potentials, but is usually rather small.

Nevertheless this behaviour gives serious restrictions for our gaugino condensation inspired model, since the low energy potential has a maximum (in the real direction) at the self-dual point $T=1$, if supersymmetry is broken in the $T$-direction (as is usually the case). Therefore high energy potentials whose minimum is at $T=1$ will not succeed in damping the coherent oscillations of the modulus.

Fig. 4 shows the trajectory of the modulus, where $V_{1}=C^{2} H^{2}(T-1.001)^{2} / 2$ for $C=10$ (a rather large value). It is clear that there is no damping at all, when compared to the trajectory with $T_{1}=1.7$ and $C=10$, which is also given in fig. 4 .

\subsubsection{Smooth transition between high and low energy minima}

If the minimum of $V_{1}$ does not lie close to a maximum of $V_{0}$ there will be a smooth transition of the minimum to its final value. In 9] it was shown that for parabolic $V_{0}$ and $V_{1}$ this can lead to a substantial damping of the coherent modulus oscillations if $C^{2}$ is large enough.

In this section we study this damping in more detail for the gaugino condensation inspired potential with the minimum at $T=1.23$ (and at the dual point $T=0.81$ ). We assume the high energy potential $V_{1}$ to be of parabolic form $V_{1}=C^{2} H^{2}\left(T-T_{1}\right)^{2} / 2$. 


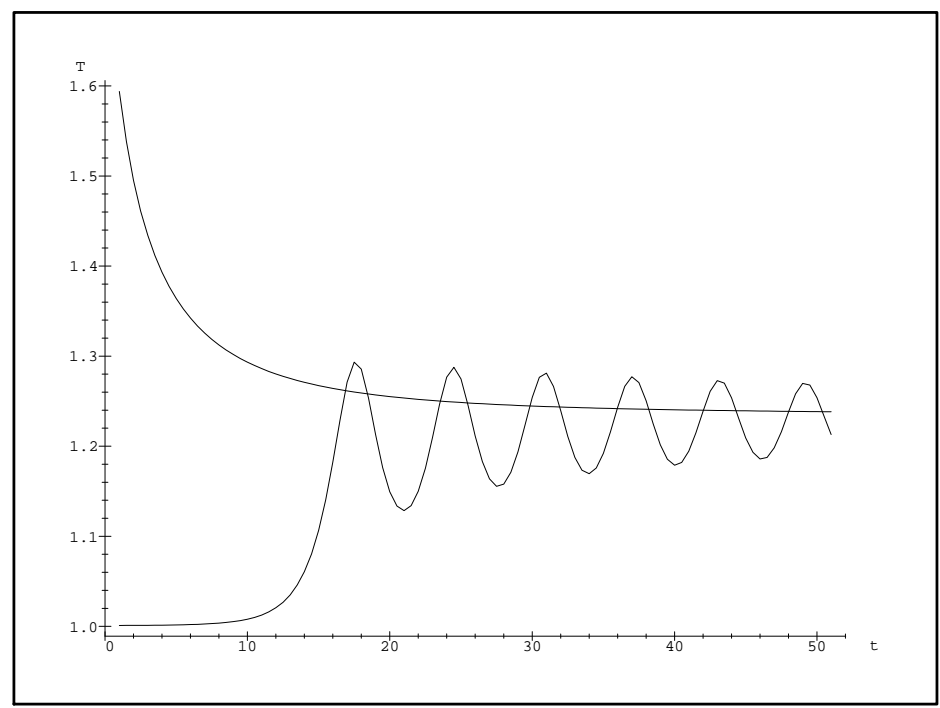

Fig 4: $T$-trajectory for $T_{1}=1.001$ and $T_{1}=1.7$ for $C=10$

Unfortunately it is impossible to repeat the analytical approach of [9] to get the damping of the oscillation amplitude. This is, because the more realistic potentials we are interested in do not have the simple quadratic structure but contain the Dedekind function $\eta$ and its derivatives.

Trying to approximate the model by an expansion which can be solved analytically proved to be inconsistent with the numerical solution because the terms one has to omit become too large. Therefore we had to stick to numerical simulations.

We have studied the dependence of the final oscillation amplitude for a set of different starting values. The results are given in figs. 5 and 6. Each curve represents a number of runs with the same initial minimum $T_{1}$ (given by the label at the curve) and shows the resulting (normalized) amplitude for different values of $C$ on a logarithmic scale. The curve with the label 'Linde' represents the $C$-dependence of the oscillation amplitude of the model of [9].

It is clear from these results that the damping of the moduli oscillations does not only depend on the value of $C$, but also strongly on the shape of the potential $V_{0}$ between the minima of $V_{0}$ and $V_{1}$. If $T_{1}$ is close to the final minimum, $V_{0}$ can be approximated by a quadratic function. As expected, the reduction of the amplitude with respect to $C$ is identical to the result in [9]. However, outside of that region, there are strong differences. If $T_{1}$ falls into a region of $V_{0}$ which is rather flat, then increasing the value of $C$ decreases the oscillation amplitude by a factor which is significantly smaller. If the modulus starts at a point where $V_{0}$ is steep, then the reduction factor is larger. 


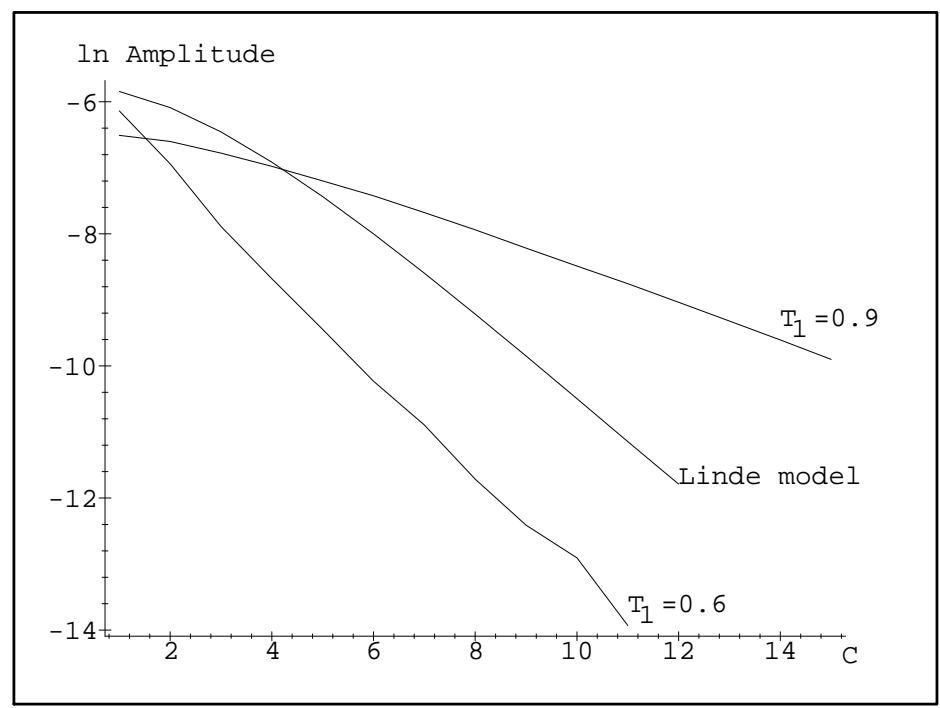

Fig 5: Damping of oscillation amplitude in comparison with model of [9]

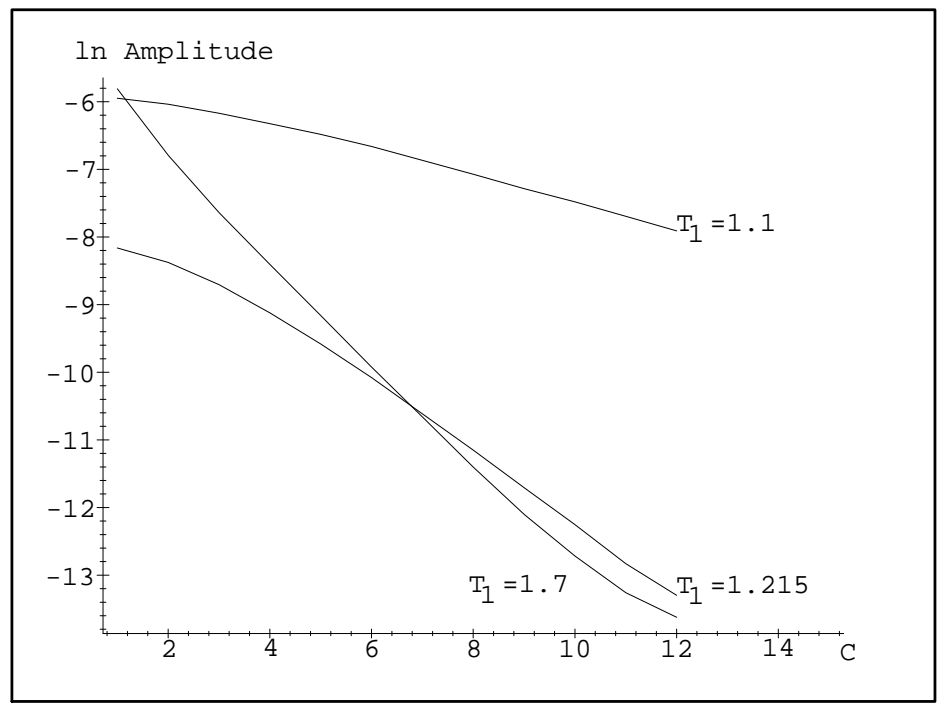

Fig 6: Damping of oscillation amplitude for additional starting values

These simulations consider $C$ to be independent of $T$. However, as we showed in the last section, $C$ will usually depend on $T$. It is therefore necessary to study the running of moduli with a non-constant $C$ and one can expect that at least for $T$ being in the vicinity of the low energy minimum, $C$ should be large.

The analysis confirms this: we choose $T_{1}=1.7$ as the high energy minimum (starting value) and allowed $C$ to vary quadratically from 1 to 8 between this initial and the final minimum like $C(T)=285(T-1.7)^{2}+1$ and $C(T)=285(T-1.23)^{2}+1$. Having $C=8$ at $T=1.7$ and $C=1$ at $T=1.23$ (the second case) results in negligible damping of the modulus oscillation. The final amplitude is even larger than for constant $C=1$ although over the whole trajectory of $T$ the value of $C$ is larger. 
The first choice, where $C$ becomes large as $T \rightarrow 1.23$, does show a damping. It corresponds however not to the maximum value of $C$ along the trajectory but to some average. The final amplitude is comparable to the case of constant $C \approx 2.5$ for this example.

To achieve significant damping it is therefore a necessary condition to have a large value of $C$ around the final minimum $T_{0}$, and a consistently large $C$ over the whole trajectory of the modulus $T$ would be sufficient.

\subsection{Explicit examples for a smooth transition}

As we have shown in the preceding sections, a model with sufficient modulus damping necessarily will fulfill two criteria: first, the high energy minimum $T_{1}$ must not be close to a maximum of $V_{0}$ and second, $C^{2}\left(T_{0}\right)=C_{0}^{2}$ must be large.

In addition it would simplify the analysis considerably if the trajectory of the modulus would be along the real axis. In principle this is not an unnatural requirement, since the theory obeys a symmetry $T \rightarrow \bar{T}$ and thus both $V_{0}$ and $V_{1}$ have an extremum on the real axis in the imaginary direction. This symmetry constitutes another example of how a symmetry of the theory could correlate the minima of the low and high energy potentials. Again the crucial point is to ensure that these extrema are indeed minima in the imaginary direction.

Unfortunately within the specified class of theories we did not succeed in constructing such a 'real' model, which has large values of $C^{2}(T)$. However, models with $T$ confined to the real axis and small $C^{2}\left(C^{2}<1\right)$ can be constructed. One example is $F(T)=$ $M_{\Phi} /\left(\eta^{6}(T) j^{1 / 6}(T)\right)$.

Relaxing the condition for $T$ to be real at all times we therefore attempt to construct models with large $C^{2}$ and complex trajectory of $T$. Furthermore we concentrate to on large final values $C_{0}^{2}$ (the necessary condition), to get an impression of the size of $C$ that can be expected.

Using the Yukawa-couplings (29) one gets for $C_{0}^{2}$ at the two minima $T_{0}=0.81,1.23$ (for broken supersymmetry)

$$
\begin{aligned}
C^{2}(1.23) & =\frac{1}{q} \frac{1}{1.23-T_{1}}(-0.63 n+4.49 l+1.55) \\
C^{2}(0.81) & =\frac{1}{q} \frac{1}{0.81-T_{1}}(-0.27 n-6.78 l+1.33) .
\end{aligned}
$$

One can see, that these models are in principle able to explain large values of $C^{2}$, which is due to the properties of the modular functions (especially $j(T)$ ). The powers of the modular functions might however be constrained by the fact that $V_{1}$ should have a minimum at finite $T$. This is ensured if $F(T) \rightarrow \infty$ for $T_{R} \rightarrow \infty$. This in turn gives the condition

$$
l>\frac{q}{12} n-\frac{w}{12} .
$$


One model which falls into this allowed region (modular weight $n=-4$ ) is then

$$
F(T)=\frac{M_{\Phi}}{\eta^{22}(T) j^{2 / 3}(T)},
$$

with $C^{2}(0.81) \simeq 7 /\left(0.81-T_{1}\right) / q$.

This is about as high as one can go if one stays within the orbifold limits on the modular weight (30) and (46).

\section{Summary and conclusion}

Our paper discusses the behaviour of string moduli during the preheating period, the time after inflation when the energy density is dominated by the coherent oscillations of $\Phi$.

We show that $\Phi$ can usually be integrated out to give an effective potential for the modulus, with a crucial dependence on the Yukawa coupling.

For this paper we restrict ourselves to models which obey $T$-duality. As the low energy potential we use the one given by the standard gaugino condensation picture. For these models it is found that a stabilization of the modulus at high energy is possible for a limited range of the modular weight $n$ of $\Phi(n<3)$ only. Within this limit, the reduction of the initial modulus amplitude around the high energy minimum can be calculated and will be $10^{5}$ at most. To be compatible with standard nucleosynthesis one would need a reduction factor of about $10^{10}$.

Studying the further time evolution of the modulus, we consider the different possibilities of how the modulus can settle to its low energy minimum.

First we construct explicit examples with coinciding low and high energy minima at the self-dual points. In this case the initial stabilization will survive the subsequent evolution. Nevertheless the reduction is not large enough to completely solve the cosmological moduli problem, if the initial amplitude (at the end of inflation) is of the order 1 (in Planck units). One therefore needs a pre-stabilization by coinciding minima during inflation and preheating as well.

Even then quantum fluctuations during inflation will drive the modulus away from its minimum, so that the additional reduction during preheating might be crucial to solve the moduli problem.

A stabilization at self-dual points implies unbroken supersymmetry in the direction of the respective modulus. It can thus not work for all moduli, if supersymmetry is broken by a modulus at all. Another mechanism to avoid modulus oscillations, namely the smooth transition from high to low energy minimum (induced by a mass term $C^{2} H^{2}$ with large $C)$ is studied next.

We found the modulus evolution in a more realistic ( $\mathrm{T}$-dual) potential to be more complicated than in a quadratic potential. If the high energy minimum is close to a low energy maximum the modulus oscillation is not significantly damped, irrespectively of the value of $C^{2}$. This might be quite relevant, since the usual $\mathrm{T}$-dual potential for broken supersymmetry has a saddle at the self-dual point $T=1$. 
The reduction factor will also depend on the shape of the low energy potential along the trajectory of $T$. If $V$ is steep along this trajectory, the reduction will be larger for the same values of $C$.

A nice feature of our framework is that it allows us to calculate $C$ from the model. Large values of $C$ are directly linked to steep couplings (large gradient with respect to $T)$.

Finally we analyze specific models and calculate the respective values of $C(T)$. Our experience shows that it is difficult to construct examples which fulfill all requirements, namely: a well defined high energy minimum, a large value of $C^{2}(T)$ along the whole trajectory and (for convenience) a trajectory along the real axis. Though modular functions like $j(T)$ are well suited to produce steep couplings, we could not obtain values of $C^{2}$ larger than $O(10)$. We believe that this in the upper region of what one can achieve. This can be contrasted to the values of $C^{2}$ needed to solve the moduli problem, which have to be at least one or two orders of magnitude larger.

We believe that our work has serious implications for the cosmological moduli problem. The additional stabilization of the modulus at its high energy minimum during preheating will provide a large amplitude reduction which can help in solving the moduli problem. As we have shown, a further damping of the oscillation can be achieved via the two possibilities of coinciding minima and smooth transitions. For the second the oscillation amplitude will be governed by the factor $C^{2}(T)$ and the shape of the potential. However, it seems unlikely that the amplitude can be reduced down to the nucleosynthesis bound. Nevertheless, given some additional small reduction (e.g. by weak scale inflation), one might be able to achieve this. In any case these results can significantly lessen the demands on other mechanisms.

Acknowledgment We thank Stephan Stieberger for information about Yukawa-couplings in string theory. This work was partially supported by the EC under contract no. SC1CT92-0789 and by the Sonderforschungsbereich 375-95 'Research in Astroparticlephysics' of DFG.

\section{References}

[1] B. de Carlos, J. A. Casas, F. Quevedo and E. Roulet, Phys. Lett. B 318 (1993) 447; T. Banks, D. B. Kaplan and A. E. Nelson, Phys. Rev. D 49 (1994) 779.

[2] G. D. Coughlan, W Fischler, E. W. Kolb, S. Raby and G. G. Ross, Phys. Lett. B 131 (1983) 59.

[3] T. Banks, M. Berkooz and P. J. Steinhardt, Phys. Rev. D 52 (1995) 705; G. G. Ross and S. Sarkar, Successful Supersymmetric Inflation, CERN-TH.95/134, hep$\mathrm{ph} / 9506283$.

[4] L. Randall and S. Thomas, Nucl. Phys. B 449 (1995) 229. 
[5] D. H. Lyth and E. D. Stewart, Phys. Rev. D 75 (1995) 201; Phys. Rev. D 53 (1996) 1784.

[6] M. Dine, W. Fischler and D. Nemeschansky, Phys. Lett. B 136 (1984) 169; G.D. Coughlan, R. Holman, P. Ramond and G.G. Ross, Phys. Lett. B 140 (1984) 44; A.S. Goncharov, A.D. Linde and M.I. Vysotsky, Phys. Lett. B 147 (1984) 279; G. Dvali, Inflation versus the Cosmological Moduli Problem, IFUP-TH-09-95, hep-ph/9503259; M. Dine, L. Randall and S. Thomas, Nucl. Phys. B 458 (1996) 291.

[7] M. Dine, L. Randall and S. Thomas, Phys. Rev. Lett. 75 (1995) 398.

[8] R. Brustein and P. J. Steinhardt, Phys. Lett. B 302 (1993) 196;

[9] A. Linde, Relaxing the Cosmological Moduli Problem, SU-ITP-96-03, hep-th/9601083.

[10] J. Ellis, J. E. Kim and D. V. Nanopoulos, Phys. Lett B 145 (1984) 181; R. Juskiewicz, J. Silk and A. Stebbins, Phys. Lett B 158 (1985) 463; J. Ellis, D. V. Nanopoulos and S. Sarkar, Nucl. Phys. B 219 (1985) 175; M. Kawasaki and T. Moroi, Prog. Theor. Phys. 93 (1995) 879.

[11] H. P. Nilles, Phys. Lett. B 115 (1982) 193; S. Ferrara, L. Girardello and H. P. Nilles, Phys. Lett. B 125 (1983) 457; J. P. Derendinger, L. E. Ibáñez and H. P. Nilles, Phys. Lett. B 155 (1985) 467; M. Dine, R. Rohm, N. Seiberg and E. Witten, Phys. Lett. B 156 (1985) 55; T.R. Taylor, Phys. Lett. B 164 (1985) 43.

[12] A. D. Linde, Phys. Lett. B 108 (1982) 389; A. Albrecht and P. J. Steinhardt, Phys. Rev. Lett 48 (1982) 1220.

[13] A. D. Linde, Phys. Lett. B 249 (1990) 18; Phys. Lett. B 259 (1991) 38; Phys. Rev. D 49 (1994) 748.

[14] L. Dixon, D. Friedan, E. Martinec and S. Shenker, Nucl. Phys. B 282 (1987) 13; S. Hamidi and C. Vafa, Nucl. Phys. B 279 (1987) 465; J. Erler, D. Jungnickel, M. Spaliński and S. Stieberger, Nucl. Phys. B 397 (1993) 379; J. A. Casas, F. Gómez and C. Muñoz, Int. J. Mod. Phys. A8 (1993) 455.

[15] S. Ferrara, D. Lüst, A. Shapere and S. Theisen, Phys. Lett. B 225 (1989) 363; J. Lauer, J. Mas and H. P. Nilles, Phys. Lett. B 226 (1989) 251; Nucl. Phys. B 351 (1991) 353; E. J. Chun, J. Mas, J. Lauer and H. P. Nilles, Phys. Lett. B 233 (1989) 141; S. Ferrara, D. Lüst and S. Theisen, Phys. Lett. B 233 (1989) 147.

[16] P. Candelas, X. de la Ossa, A. Font, S. Katz and D. R. Morrison, Nucl. Phys. B416 (1994) 481; S. Hosono, A. Klemm, S. Theisen and S. T. Yau, Comm. Math. Phys. 167 (1995) 301; Nucl. Phys. B 433 (1995) 501; A. Klemm, W. Lerche and P. Mayr, Phys. Lett. B 357 (1995) 313; V. Kaplunovsky, J. Louis, S. Theisen, Phys. Lett. B 357 (1995) 71. 
[17] L. E. Ibáñez and D. Lüst, Nucl. Phys. B 382 (1992) 305.

[18] A. Font, L. E. Ibáñez, D. Lüst and F. Quevedo, Phys. Lett. B 245 (1990) 401; H. P. Nilles and M. Olechowski, Phys. Lett. B 2481990 268; P. Binetruy and M.K. Gaillard, Phys. Lett. B 253 (1991) 119; M. Cvetic, A. Font, L. E. Ibáñez, D. Lüst and F. Quevedo, Nucl. Phys. B 361 (1991) 194.

[19] R. Brustein and P. J. Steinhardt, Phys. Lett. B 302 (1993) 196. 